\title{
Efficient Evaluation of the Accuracy of Molecular Quantum Dynamics on an Approximate Analytical or Interpolated Ab Initio Potential Energy Surface
}

\author{
TOMÁŠ ZIMMERMANN, ${ }^{1}$ JULIEN RUPPEN, ${ }^{1}$ BAIQING LI, ${ }^{1,2}$ \\ JIŘí VANÍČEK ${ }^{1}$ \\ ${ }^{1}$ Laboratory of Theoretical Physical Chemistry, Institut des Sciences et Ingénierie Chimiques, \\ École Polytechnique Fédérale de Lausanne (EPFL), CH-1015 Lausanne, Switzerland \\ ${ }^{2}$ School of Chemistry and Chemical Engineering, Shandong University, Jinan, Shandong 250100, \\ People's Republic of China
}

Received 18 December 2009; accepted 11 March 2010

Published online 10 June 2010 in Wiley InterScience (www.interscience.wiley.com).

DOI 10.1002/qua.22730

\begin{abstract}
Ab initio electronic structure methods have reached a satisfactory accuracy for the calculation of static properties, but remain too expensive for quantum dynamical calculations. Recently, an efficient semiclassical method was proposed to evaluate the accuracy of quantum dynamics on an approximate potential without having to perform the expensive quantum dynamics on the accurate potential. Here, this method is applied for the first time to evaluate the accuracy of quantum dynamics on an approximate analytical or interpolated potential in comparison to the quantum dynamics on an accurate potential obtained by an ab initio electronic structure method. Specifically, the vibrational dynamics of $\mathrm{H}_{2}$ on a Morse potential is compared with that on the full CI potential, and the photodissociation dynamics of $\mathrm{CO}_{2}$ on a LEPS potential with that on the excited ${ }^{1} \Pi$ surface computed at the EOM-CCSD/aug-cc-pVDZ level of theory. Finally, the effect of discretization of a potential energy surface on the quantum dynamics is evaluated. (ㄱ) 2010 Wiley Periodicals, Inc. Int J Quantum Chem 110: 2426-2435, 2010
\end{abstract}

Key words: quantum dynamics; quantum fidelity; ab initio method; potential energy surface; semiclassical 


\section{Introduction}

A $\mathrm{b}$ initio methods for the electronic structure of molecules have reached a satisfactory accuracy for the calculation of static properties, such as energy barriers or force constants at local minima of the potential energy surface (PES). The most accurate of such methods remain out of reach when one wants to describe molecular properties that depend on full quantum dynamics [1]. To make a calculation feasible, one has to approximate either the dynamics of the system [2] or the PES [1], but both approaches can have nontrivial effects on the results [3].

A recent paper [4] has proposed an efficient and accurate way to determine the consequences of approximating the PES. Namely, the authors have considered quantum dynamics that is done exactly but on a PES obtained by a lower level electronic structure method that is less accurate but also less expensive [4]. When such a calculation is finished, its accuracy is not known because of the forbidding expense of the dynamics on the more accurate potential. Li, Mollica, and Vaníček have demonstrated the feasibility of their approach by analyzing the effect of perturbation of an analytical LEPS potential $[5,6]$ for the photodissociation of $\mathrm{CO}_{2}$. Here, for the first time, we show how the approach from Ref. [4] can be used to assess the accuracy of an analytical PES in comparison with an accurate, truly ab initio PES. In particular, we show that the quantum dynamics and resulting observables can be quite different even if the difference between the two PESs is quite small in the dynamically accessible region. We can estimate the error of the quantum dynamics on the analytical potential without computing the dynamics on the $\mathrm{ab}$ initio potential.

In addition to being computed by less accurate methods, a PES used for the quantum dynamics is often computed only on a grid which is sparser than the grid used in the actual quantum dynamics. The potential energy at grid points which lie "in between" are then obtained by some interpolation method. The above approach used to evaluate the accuracy of the approximate PES can also be employed to evaluate the accuracy of the interpolated PES with respect to the PES computed on-thefly. With this ability, our method can be used as a fast tool to find an appropriate grid density necessary to achieve a desired accuracy.

The remainder of the article is organized as follows: In Section 2, we describe the dephasing representation of quantum fidelity and how it is used to evaluate the accuracy of a PES. In Section 3, the methodology is tested on the vibrational dynamics of the $\mathrm{H}_{2}$ molecule, where we compare an analytical Morse potential with an ab initio potential at the full CI/cc-pVTZ level of theory, and on the photodissociation dynamics of $\mathrm{CO}_{2}$, where we compare the analytical LEPS potential with an ab initio potential at the EOM-CCSD/aug-cc-pVDZ level. We also evaluate the effect of constructing the PES by interpolation from a sparser ab initio computed potential energy grid. Section 4 concludes the article.

\section{Methodology}

For simplicity, we use the Born-Oppenheimer approximation and focus on the quantum dynamics of nuclei, although the method of Ref. [4] applies to any quantum dynamics and has been used successfully to analyze the importance of nonadiabatic effects [7]. The molecular time-dependent Schrödinger equation is solved in two stages: First, the time-independent equation for electrons is solved with fixed nuclear configurations, and then the nuclear motion is calculated on the resulting electronic PES. We consider two PESs: $V_{\text {acc }}$ is a very accurate high-level electronic structure PES (presumably "almost exact"), which is too expensive to be used for quantum dynamics. $V_{\text {appr }}$ is an approximate PES, obtained by a lower-level electronic structure method or by an analytical fit of $V_{\text {acc, }}$ and "cheap" enough to be used for quantum dynamics.

In Ref. [4], the accuracy of quantum dynamics on the approximate PES is quantified by computing the quantum-mechanical (QM) overlap

$$
f_{\mathrm{QM}}(t):=\left\langle\psi_{\mathrm{acc}}(t) \mid \psi_{\mathrm{appr}}(t)\right\rangle,
$$

where the subscript of $\psi$ denotes the corresponding PES used for the propagation of the initial state $\psi(0)$. The quantity $F(t):=\left|f_{\mathrm{QM}}(t)\right|^{2}$ is known as quantum fidelity or Loschmidt echo and has been defined by Peres [8] to measure the sensitivity of quantum dynamics to perturbations. Much effort has been devoted to the study of the temporal decay of fidelity and many universal regimes have been found [9]. In our setting, if $F(t) \approx 1$ for all times up to $t_{\max }$, we can trust quantum dynamics on the approximate potential $V_{\text {appr }}$ and use the resulting $\psi_{\text {appr }}(t)$ to compute all dynamical properties up to $t_{\max }$.

Since $V_{\text {acc }}$ is too expensive, neither $\psi_{\text {acc }}(t)$ nor $f_{\mathrm{QM}}(t)$ can be computed. Li, Mollica, and Vaníček 
have circumvented the necessity to compute $\psi_{\text {acc }}(t)$ by using the dephasing representation (DR) of quantum fidelity [10, 11]. The DR is a semiclassical approximation of fidelity [12, 13] that has been shown to be accurate in chaotic, integrable, and mixed systems even in nonuniversal regimes sensitive to the choice of the initial state and details of dynamics $[10,11]$. To evaluate the accuracy of $V_{\text {appr }}$, a specific type of "perturbation" is considered, namely the difference $\Delta V=V_{\text {appr }}-V_{\text {acc }}$ between the approximate and accurate PESs. The DR can be derived from the semiclassical initial value representation (IVR) expression for fidelity amplitude [11]

$f(t)$

$$
\begin{aligned}
= & (2 \pi \hbar)^{-2 d} \int d x_{\mathrm{appr}}^{0} \int d x_{\mathrm{acc}}^{0} \int d s\left|\frac{\partial q_{\mathrm{appr}}^{t}}{\partial p_{\mathrm{appr}}^{t}}\right|^{1 / 2}\left|\frac{\partial q_{\mathrm{acc}}^{t}}{\partial p_{\mathrm{acc}}^{t}}\right|^{1 / 2} \\
& \times \psi^{*}\left(q_{\mathrm{acc}}^{0}\right) \psi\left(q_{\mathrm{appr}}^{0}\right) \exp \left\{\frac{i}{\hbar}\left[S_{\mathrm{appr}}^{t}-S_{\mathrm{acc}}^{t}+s \cdot \Delta q^{t}\right]\right\} .
\end{aligned}
$$

Here $x$ denotes a phase-space point $(q, p)$, superscript $t$ is the corresponding time, $x_{\mathrm{appr}}^{t}$ and $x_{\mathrm{acc}}^{t}$ denote the trajectories on the approximate and accurate PESs, respectively. Finally, $S_{\text {appr }}^{t}$ and $S_{\text {acc }}^{t}$ are the corresponding actions. To simplify the evaluation of this highly oscillatory integral, the shadowing theorem [14] is invoked, which states that for every trajectory on the accurate PES, a very near ("shadowing") trajectory on the approximate PES exists. Using the shadowing trajectories, the action $S_{\text {acc }}^{t}$ can be linearly expanded about $S_{\text {appr }}^{t}$. The stationary phase approximation yields the DR as an interference integral

$$
\begin{aligned}
f_{\mathrm{DR}}(t) & :=\int d x^{0} \rho_{\mathrm{W}}\left(x^{0}\right) \exp \left[-i \Delta S\left(x^{0}, t\right) / \hbar\right], \\
\Delta S\left(x^{0}, t\right) & =\int_{0}^{t} d \tau \Delta V\left[q_{\mathrm{appr}}^{\tau}\left(x^{0}\right)\right] .
\end{aligned}
$$

Here, $\Delta S\left(x^{0}, t\right)$ is the action due to $\Delta V$ along the trajectory $q_{\mathrm{appr}}^{t}\left(x^{0}\right)$ of $V_{\mathrm{appr}}$, and $\rho_{\mathrm{W}}$ is the Wigner function of the initial state $\psi$,

$\rho_{\mathrm{W}}(x)$

$$
=h^{-d} \int d \xi \psi^{*}\left(q+\frac{\xi}{2}\right) \psi\left(q-\frac{\xi}{2}\right) \exp \left(i \frac{\xi \cdot p}{\hbar}\right) .
$$

In the "dephasing representation," all of fidelity decay appears to be due to interference and none due to the decay of classical overlaps. By using only the trajectories of the approximate PES, the dephasing representation is related to the semiclassical perturbation approximation [15]. However, in our case, due to the shadowing property, it is not necessary to assume that the trajectory of $V_{\text {acc }}$ remains near the trajectory of $V_{\text {appr }}$ with exactly the same initial condition $[12,13]$.

Note that we suggest using trajectories of $V_{\text {appr }}$ for computing $f_{\mathrm{DR}}$. In principle, one could instead use trajectories of $V_{\text {acc }}$ [which would only mean switching the roles of $V_{\text {acc }}$ and $V_{\text {appr }}$ in Eqs. (2) and (3)] but when compared with the exact QM result (1), the expected accuracy would be similar to the accuracy of Eq. (2). The reason for choosing expression (2) together with (3) is simply efficiency: the DR expression (2) requires values and gradients of the "cheaper" PES $\left(V_{\text {appr }}\right)$ but only values of the more expensive PES $\left(V_{\text {acc }}\right)$, whereas the dual expression based on trajectories of $V_{\text {acc }}$ would require values of $V_{\text {appr }}$ but both values and gradients of $V_{\text {acc }}$.

The fundamental reason why quantum dynamics calculations are expensive is nonlocality of quantum mechanics: Wave function $\psi(t+\Delta t)$ at any point in space depends in general on $\psi(t)$ in the whole space. Therefore, exact quantum dynamics, no matter on which PES, is only possible for few degrees of freedom (DOF). We expect the DR to be useful for evaluating the accuracy of a PES in future applications with more DOFs where the quantum dynamics itself has to be approximated in addition to approximating the PES. In this article, we aim to show the accuracy of the DR for estimating the difference between wave functions evolved on the ab initio potential $V_{\text {acc }}$ and the analytical potential $V_{\text {appr }}$. We, therefore, need the exact quantum dynamics for comparison. Consequently, we only consider systems with few DOF for which one can construct a semiglobal PES $V_{\text {acc }}$, i.e., compute a potential energy grid with $n_{j}$ points in the $j$ th DOF.

Once the PES is known, dynamics can be performed, e.g., by the second order split-operator method [16]. In this method, the quantum evolution operator for time step $\Delta t$ is approximated by the following:

$$
e^{-i \hat{H} \Delta t / \hbar}=e^{-i \hat{V} \Delta t / 2 \hbar} e^{-i \hat{T} \Delta t / \hbar} e^{-i \hat{V} \Delta t / 2 \hbar}+O\left(\Delta t^{3}\right),
$$

where $\hat{H}=\hat{T}+\hat{V}$ is the Hamiltonian of the system and $\hat{T}$ is the kinetic energy operator. Quantum dynamics consists of alternate kinetic and potential propagations (which are just multiplications in 
appropriate representations) and a fast Fourier transform (FFT) to change the representation in between. The complexity of the FFT is $O(N \log N)$ where $N=$ $\prod_{j=1}^{d} n_{j}$ is the dimension of the Hilbert space and $d$ is the number of DOF. The overall cost of quantum propagation is $O\left(d t n^{d} \log n\right)$ where $n:=N^{1 / d}$ is the average number of grid points per DOF.

In the DR, potential energy and forces are evaluated on-the-fly, along classical trajectories. This makes the DR calculation feasible, like classical molecular dynamics (MD), for very large systems. The costs of evaluating $V_{\text {appr }}$ and $\nabla V_{\text {appr }}$ are very small since we have an analytical expression for $V_{\text {appr }}$. The cost of evaluating $V_{\text {acc }}$ along the classical trajectories is even smaller since we can take advantage of the potential energy grid $V_{\text {acc }}$ constructed for the quantum dynamics. While the classical trajectory is unlikely to go exactly through the precomputed grid points, we can easily obtain the value of $V_{\text {acc }}$ at the point $q_{\mathrm{appr}}^{t}$ by interpolation on-the-fly. (We have used multilinear interpolation [17].) The overall cost of the DR dynamics is $O\left(d n_{\text {paths }} t\right)$, where the number of trajectories needed, $n_{\text {paths }}=C(F) \sigma^{-2}$ is approximately independent of $d, t$, or the type of classical dynamics, and only depends on the value of fidelity one wants to describe and the statistical error one wants to achieve [4]. We have assumed that the dominant cost at each step is the evaluation of the force $\nabla V_{\text {appr }}$ and that it is proportional to $d$ : Such scaling with $d$ could be attained, e.g., by using a finite difference approach. In particular, in the DR, there is no exponential scaling with the number of DOF.

For the propagation of trajectories and computing the classical action $\Delta S$ according to the DR expression (3), we have used the Verlet algorithm [18],

$$
\begin{aligned}
p\left(t+\frac{\Delta t}{2}\right) & =p(t)-\nabla V_{\mathrm{appr}}(q(t)) \frac{\Delta t}{2}, \\
q(t+\Delta t) & =q(t)+m^{-1} p\left(t+\frac{\Delta t}{2}\right) \Delta t, \\
p(t)+\Delta t) & =p\left(t+\frac{\Delta t}{2}\right)-\nabla V_{\mathrm{appr}}(q(t+\Delta t)) \frac{\Delta t}{2},
\end{aligned}
$$

which is the second order symplectic integrator corresponding exactly to the split operator method (5) for quantum dynamics.

In general, the quantum wave function can be much less smooth than the PES. Recall, e.g., the wave function of the $n$th excited state of the simple harmonic oscillator which has $n$ local minima, whereas the potential has only one minimum. Quantum dynamics, however, requires the knowledge of the propagator, and therefore of the potential, on the same grid as the wave function. As the ab initio potential $V_{\text {acc }}$ is very expensive, it is convenient to perform the electronic structure calculations on a much sparser grid of $\prod_{j=1}^{d} \bar{n}_{j}$ points with $\bar{n}_{j} \ll n_{j}$ and then construct the dense PES grid for quantum dynamics by interpolation from the sparse grid. Again, we have used the multilinear interpolation [17]. In fact, the effect of interpolation can be analyzed by the DR and this is done in Section 3.3.

\section{Results}

\subsection{VIBRATIONAL DYNAMICS OF $\mathrm{H}_{2}$}

The first system considered was the vibrational dynamics of the $\mathrm{H}_{2}$ molecule. After removing rotations and translations, a single vibrational DOF is left: the bond length $r$. As $\mathrm{H}_{2}$ is a 2-electron molecule, the full CI PES $V_{\text {acc }}=V_{\mathrm{FCI}}$ can be computed using the correlation consistent polarized valence triple zeta basis set (cc-pVTZ) [19]. For the approximate analytical potential $V_{\text {appr }}$, we have used the Morse potential [20],

$$
V_{\text {Morse }}(r):=V_{0}+D_{\mathrm{e}}\left(1-e^{-\left(r-r_{0}\right) / a}\right)^{2} .
$$

Above, $V_{0}$ is the potential energy at the minimum, $D_{\mathrm{e}}$ is the classical dissociation energy, $r_{0}$ is the equilibrium bond length, and $a$ controls the steepness of the potential. Values of $V_{0}, r_{0}, D_{\mathrm{e}}$, and $a$ were fitted to $V_{\text {acc }}$ via the least squares method in the bond length range 0.79 a.u. $<r<4.26$ a.u. The resulting parameter values were $V_{0}=-1.1737$ a.u., $r_{0}=1.4279$ a.u., $D_{\mathrm{e}}=0.1947$ a.u., and $a=1.0052$ a.u. The two potentials, $V_{\text {acc }}$ and $V_{\text {appr }}$, are compared in Figure 1. In the dynamically accessible region, $\left|V_{\text {acc }}-V_{\text {appr }}\right| \leq 9.0 \times 10^{-3}$ a.u.

Both DR and QM dynamics calculations were performed for $2000 \mathrm{fs}$ with a time step of 2 a.u. $\approx 0.05$ fs on a grid with $n=2048$ points $6 \times 10^{-3}$ a.u. apart. The full CI/cc-pVTZ PES grid $V_{\text {acc }}=V_{\mathrm{FCI}}$ was computed with Gaussian 09 [21]. Thanks to the low cost of the ab initio potential $V_{\mathrm{FCI}}$, the construction of the PES did not require interpolation. Interpolation was only used for evaluating $V_{\text {acc }}$ along the $n_{\text {paths }}$ classical trajectories used in the DR calculation. We have used $n_{\text {paths }}=512$ which should result in a statistical error smaller than $n_{\text {paths }}^{-1 / 2} \approx 4 \%$, but to confirm this, we reran calculations with $n_{\text {paths }}=16,384$ 

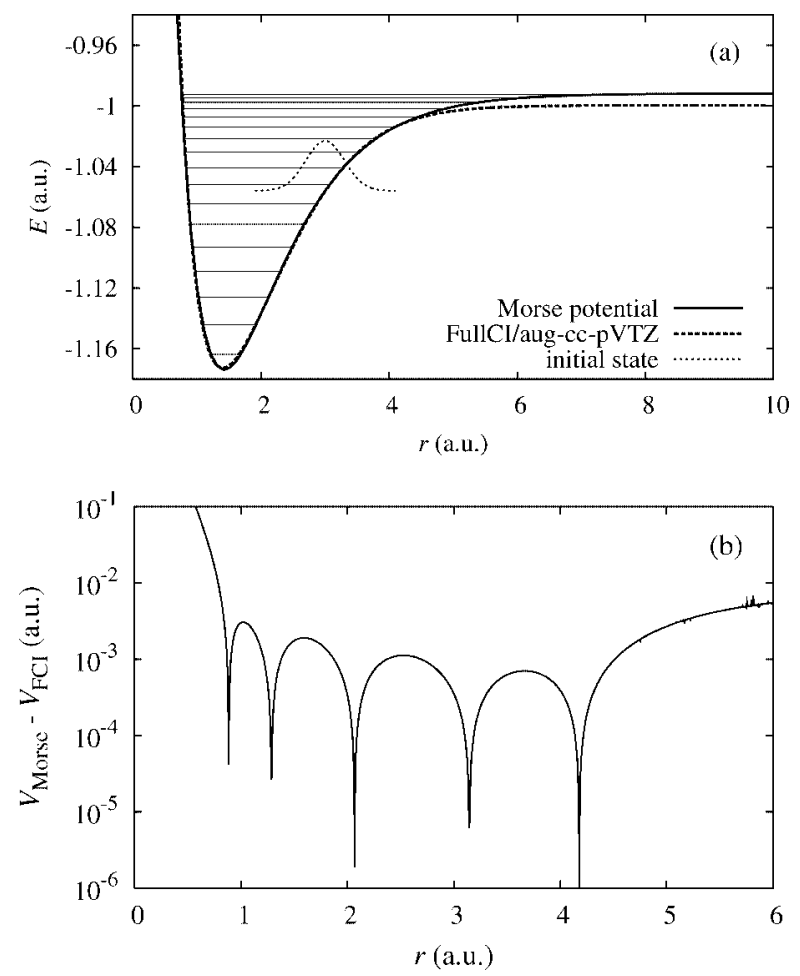

FIGURE 1. (a) Comparison of $V_{\text {appr }}=V_{\text {Morse }}$ and $V_{\text {acc }}=V_{\mathrm{FCl}}$ for the $\mathrm{H}_{2}$ molecule. Also shown are the vibrational energy levels of $V_{\text {Morse }}$ and the initial wave packet. (b) The difference $\left|V_{\text {appr }}-V_{\text {acc }}\right|=\left|V_{\text {Morse }}-V_{\text {FCI }}\right|$ between the two potentials as a function of the bond length.

and $n_{\text {paths }}=131,072$. Also, $n_{\text {paths }}=64$ was used, to demonstrate that even with a much lower number of paths a good estimate of fidelity can be obtained. The initial state was a Gaussian wave packet, with position and momentum centered at $R_{0}=3$ a.u., $P_{0}=0$ a.u., respectively, and with a position width $\sigma_{r}=0.3$ a.u.

Figure 2(a) shows the fidelity of the vibrational dynamics on the Morse potential (7) in comparison to $V_{\mathrm{FCI}}$ for the time of $50 \mathrm{fs}$ which contains several vibrational periods since we expect that the main features of the spectrum will be resolved by this time. First of all, the figure confirms that the fidelity can be computed accurately by the DR since the DR and exact $Q M$ results agree very well. In other words, we can estimate the accuracy of quantum dynamics on $V_{\text {Morse }}$ by $F_{\mathrm{DR}}$ without having to perform quantum dynamics on $V_{\mathrm{FCI}}$ which is needed for computing $F_{\mathrm{QM}}$. Second, by comparing the DR results for 64; 512; 16,384; 131,072 trajectories, Figure 2(a) shows that even using 64 trajectories suffices to obtain an adequate estimate of fidelity. Third, the figure shows that for our system, the fidelity remains close to unity $(>0.9)$ in the given range of time, and therefore, we expect that the dynamical observables dependent on the dynamics up to time $t_{\max }=50 \mathrm{fs}$ to be accurately described by using $V_{\text {Morse }}$. We emphasize that this conclusion would be hard to predict from the static comparison of the potentials in Figures $1(\mathrm{a})$ and (b). An example of an observable is the vibrational spectrum that can be obtained simply by taking the Fourier transform of the autocorrelation
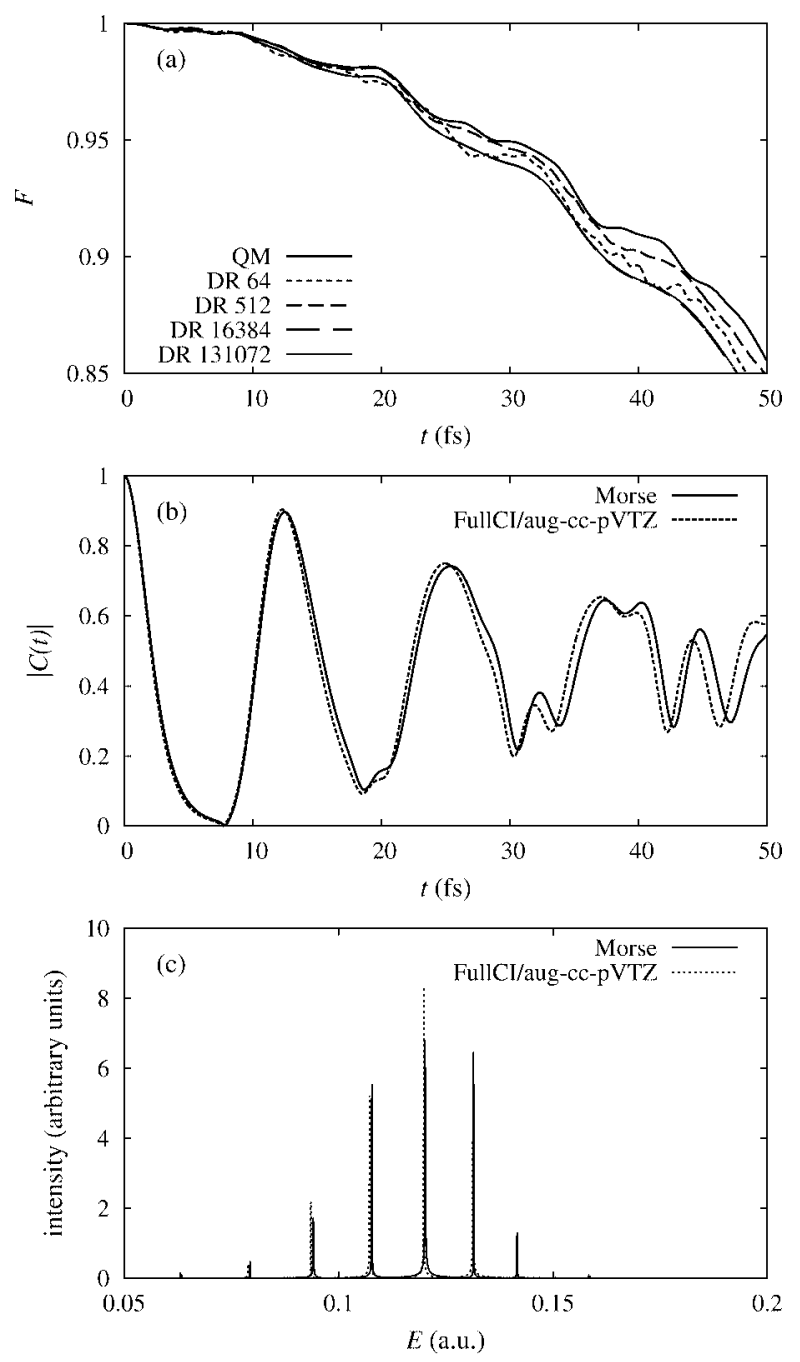

FIGURE 2. Comparison of vibrational quantum dynamics of $\mathrm{H}_{2}$ on $V_{\text {Morse }}$ and $V_{\mathrm{FCl}}$. The initial state was a Gaussian wave packet with $R_{0}=3$ a.u., $P_{0}=0$, and $\sigma_{r}=0.3$ a.u. (a) Quantum fidelity computed by the DR and exactly (QM). (b) Comparison of the autocorrelation functions on $V_{\text {Morse }}$ and $V_{\mathrm{FCl}}$. (c) Comparison of vibrational spectra of $V_{\text {Morse }}$ and $V_{\mathrm{FCl}}$. 
function $C(t)=\langle\psi(0) \mid \psi(t)\rangle$. Figure 2(b) compares the autocorrelation functions computed using $V_{\text {acc }}$ and $V_{\text {appr }}$, and Figure 2(c) the corresponding vibrational spectra. The two spectra have noticeable but small differences, and so we can conclude that in this case, the Morse potential $V_{\text {Morse }}$ is an accurate representation of the ab initio potential $V_{\mathrm{FCI}}$. The DR has correctly predicted this without having to compute the dynamics or the vibrational spectrum on $V_{\mathrm{FCI}}$.

\subsection{PHOTODISSOCIATION DYNAMICS OF $\mathrm{CO}_{2}$}

The second system considered was the collinear photodissociation of carbon dioxide, a model that had been studied extensively by both quantumdynamical [6, 22] and semiclassical methods [23]. Invoking the Franck-Condon principle, photodissociation dynamics is the quantum dynamics of the initial state (vibrational ground state of the PES of the electronic ground state) on the dissociative excited PES. The photodissociation spectrum is obtained again by the Fourier transform of the autocorrelation function $C(t)$.

In the case of carbon dioxide, a single excited surface is not sufficient for even a qualitative calculation of the photodissociation spectrum between $200 \mathrm{~mm}$ and $120 \mathrm{~nm}$. Instead, a rather involved nonadiabatic simulation would have to be performed including at least the dissociative ${ }^{1} \Pi$ surface as well as the ${ }^{1} \Sigma$ and ${ }^{1} \Delta$ surfaces, both of which have local minima near the Franck-Condon region. Here, we concentrate on the dissociative ${ }^{1} \Pi$ excited PES, which is responsible for the high energy end of the spectral band. Namely, we explore how accurately this surface is approximated by the widely used LEPS surface $[5,6]$. The accurate ab initio ${ }^{1} \Pi$ surface $V_{\text {acc }}$, computed at the EOM-CCSD/aug-cc-pVDZ level of theory [19, 24-26], is displayed in Figure 3(a) and will be referred to as $V_{\text {EOM-CCSD }}$ below. The approximate analytical potential $V_{\text {appr }}$ is given by the analytical LEPS potential for $\mathrm{CO}_{2}[5,6]$,

$$
V_{\mathrm{appr}}\left(r_{1}, r_{2}\right):=V_{\mathrm{LEPS}}\left(r_{1}, r_{2}\right) .
$$

The difference between $V_{\mathrm{EOM}-\mathrm{CCSD}}$ and $V_{\mathrm{LEPS}}$ is plotted in Figure 3(b).

The DR and QM dynamics calculations were performed for $192 \mathrm{fs}$ with a time step of 6.5 a.u. $\approx 0.16$ fs. Converged quantum calculations required $n=$ 2, 048 points to discretize each $\mathrm{CO}$ bond length from $0.6 a_{0} \approx 0.3 \AA$ to $40.6 a_{0} \approx 21.5 \AA$, altogether using a $2,048 \times 2,048$ grid to represent the PES or the wave
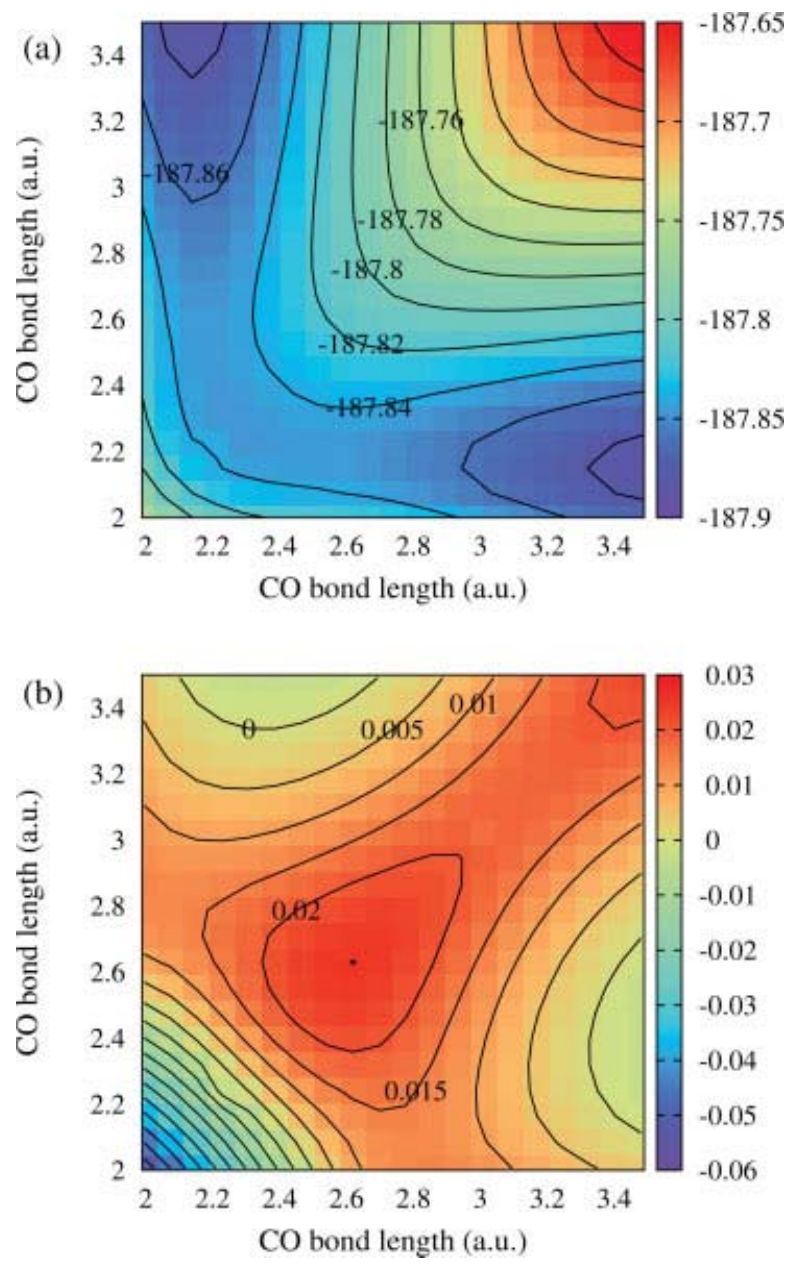

FIGURE 3. Comparison of $V_{\text {appr }}=V_{\text {LEPS }}$ and $V_{\text {acc }}=V_{\text {EOM-CCSD }}$ for the collinear configurations of $\mathrm{CO}_{2}$. (a) Contour plot of the ${ }^{1} \Pi$ surface $V_{\text {EOM-CCSD }}$. Energy is in a.u. (b) Contour plot of the difference

$\Delta V=V_{\text {EOM-CCSD }}-V_{\text {LEPS }} . \Delta V$ is set to zero in the CO + $\mathrm{O}$ dissociation limit. Energy difference is in a.u.

function. As in the case of $\mathrm{H}_{2}, n_{\text {paths }}=512$ classical trajectories were used to compute the DR of fidelity, with $n_{\text {paths }}=64 ; 16,384$; and 131,072 to confirm the convergence. The initial state was a Gaussian wavepacket corresponding to the vibrational ground state of the electronic ground state of $\mathrm{CO}_{2}$. Both $\mathrm{CO}$ bond lengths were equal to $2.212 a_{0}$. The coordinate widths of the wavepacket were $\sigma=0.075 a_{0}$ for the symmetric stretch and $\sigma=0.069 a_{0}$ for the asymmetric stretch.

The ab initio PES $V_{\text {acc }}$ was constructed by multilinear interpolation [17] of a $128 \times 128$ potential energy grid in the range from $0.6 a_{0}$ to $10.6 a_{0} \approx 5.6 \AA$ 
computed using Gaussian 09 [21]. As the dynamics in the region beyond $10.6 a_{0}$ affects neither the autocorrelation function nor the spectrum, the ab initio potential was padded by a constant asymptotic energy value in the region from 10.6 to $40.6 a_{0}$. The potential energy profiles of the ground state and the ${ }^{1} \Sigma,{ }^{1} \Delta$, and ${ }^{1} \Pi$ excited surfaces along the symmetric stretch coordinate qualitatively correspond to those obtained by Knowles et al. using the MC SCF method [27]. As the aug-cc-pVDZ basis set used to compute the ${ }^{1} \Pi$ surface is still relatively small, the accuracy of the results was checked by comparing the three lowest excitation energies at the CCSD/augcc-pVDZ minimum structure with those obtained by the same method but using the aug-cc-pVTZ and aug-cc-pVQZ basis sets.

The excitation energy of the ${ }^{1} \Pi$ state, computed with the DZ, TZ, and QZ basis sets, is, respectively, $9.07,9.02$, and $9.03 \mathrm{eV}$. In the same order, the basis dependent excitation energies for the lower lying ${ }^{1} \Sigma$ state are $9.00,8.95$, and $8.96 \mathrm{eV}$, and for the ${ }^{1} \Delta$ state, $8.79,8.90$, and $8.96 \mathrm{eV}$. This suggests that for the ${ }^{1} \Pi$ and ${ }^{1} \Sigma$ surfaces, the TZ basis set result is already converged within $10^{-2} \mathrm{eV}$ precision and that even with the DZ basis set one can obtain accurate results. Differences for ${ }^{1} \Delta$ state are higher. This is not surprising since the DZ basis set does not contain enough basis functions of the appropriate symmetry. To conclude, as we are interested only in the dynamics on the ${ }^{1} \Pi$ surface, the DZ basis seems to be sufficient to achieve the $5 \times 10^{-3}$ a.u. accuracy in the dynamically important region of the PES. This is still a twice better accuracy than that of the well-performing Morse potential used above for $\mathrm{H}_{2}$.

To compute the spectrum, the CCSD/aug-ccpVDZ zero point vibrational energies of the symmetric and asymmetric stretches were added to the CCSD/aug-cc-pVDZ ground state electronic energy in order that the zero energy reference correspond to the spectroscopic measurement. In contrast to the $a b$ initio surface, the LEPS potential is usually defined to be zero at the $\mathrm{CO}+\mathrm{O}$ dissociation limit. To compare with the ab initio spectrum, the LEPS energy had to be augmented by adding the aforementioned zero point vibrational energies as well as the electronic $\mathrm{CO}_{2} \rightarrow \mathrm{CO}+\mathrm{O}$ dissociation energy computed at the CCSD/aug-cc-pVDZ level of theory. (The basis set superposition error was corrected by the counterpoise procedure [28].)

Figure 4(a) shows the fidelity of the photodissociation dynamics on the LEPS potential in comparison to the ab initio potential. The figure confirms that the fidelity can be computed accurately by the DR
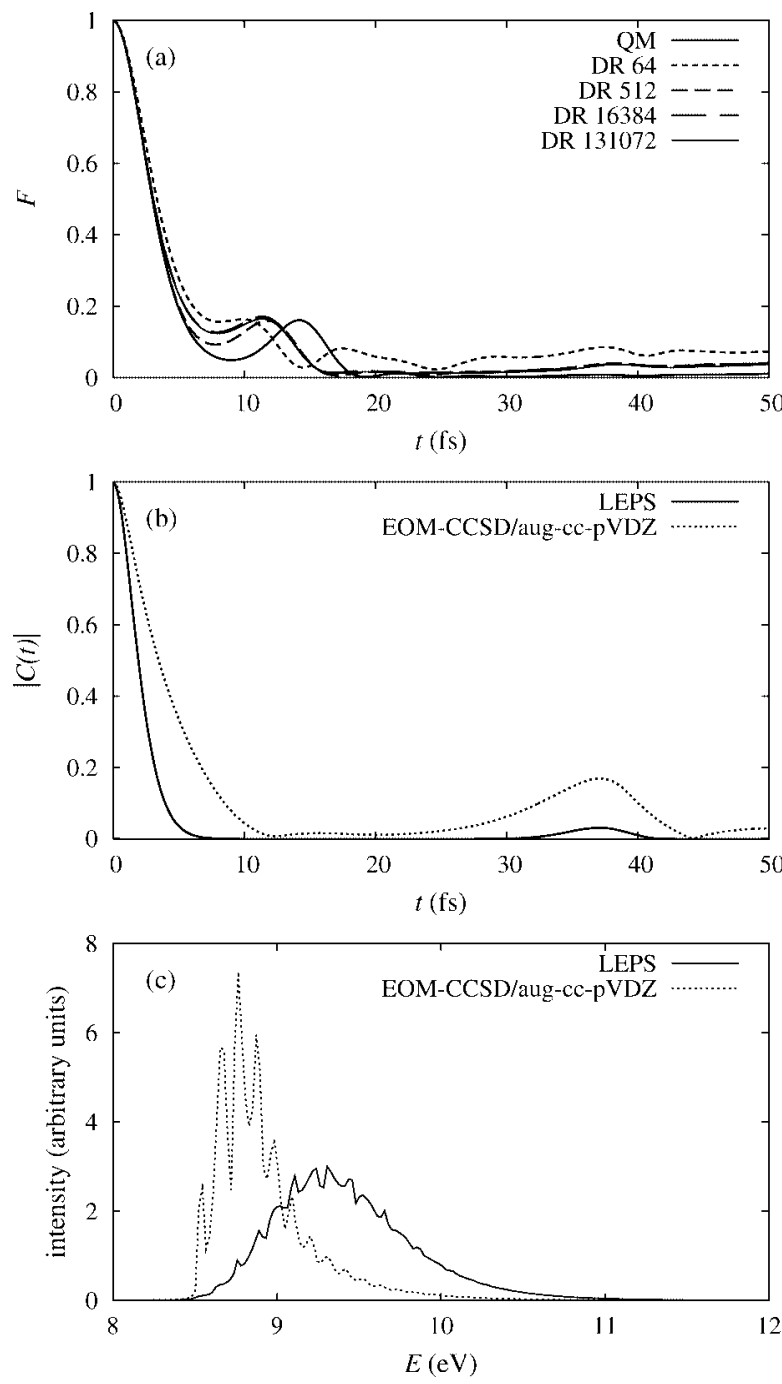

FIGURE 4. (a) Quantum fidelity of the photodissociation dynamics of $\mathrm{CO}_{2}$ on $V_{\text {LEPS }}$ and $V_{\text {EOM-CCSD }}$, computed approximately (by the DR) and exactly (QM). (b) Comparison of the autocorrelation functions on $V_{\text {LEPS }}$ and $V_{\text {EOM-CCSD }}$. (c) Comparison of the photodissociation spectra on $V_{\text {LEPS }}$ and $V_{\text {EOM-CCSD. }}$

since the DR and exact QM results agree very well up to the time where fidelity decays approximately to zero. Hence, we can avoid performing quantum dynamics on $V_{\text {EOM-CCSD. }}$.

Again, one is interested more in observables and less in the overlap of the wave functions, so we have used the dynamics to compute the photodissociation spectra on the two PESs (by a Fourier transform of the autocorrelation functions). The autocorrelation functions and photodissociation spectra corresponding to $V_{\mathrm{LEPS}}$ and $V_{\mathrm{EOM}-\mathrm{CCSD}}$ are compared 
in Figures 4(b) and (c). The two spectra are very different: they differ both in the intensities and in the positions of peaks. The DR has correctly predicted this conclusion without having to compute the photodissociation spectrum on $V_{\mathrm{EOM}-\mathrm{CCSD}}$.

In comparison with experimental spectra [29, 30] whose high energy region should be approximated by the computed spectra, we notice that the shape of this spectral region, with high and well defined peaks, is better approximated by the dynamics on the ab initio surface. The LEPS spectrum has too small peaks and is very irregular. On the other hand, the LEPS spectrum reproduces almost exactly the position of the maximum of this part of the spectrum, which is located at $\sim 9.3 \mathrm{eV}$, whereas the EOM-CCSD/aug-cc-pVDZ spectrum underestimates the position of maximum by $\sim 0.5$ $\mathrm{eV}$. It should be stressed that the comparison with experimental spectra can be qualitative at best, as the model does not consider other electronic surfaces, nonadiabatic couplings between them, the two bending vibrations, or the excited vibrational states of $\mathrm{CO}_{2}$.

\subsection{VIBRATIONAL DYNAMICS OF $\mathrm{H}_{2}$ ON AN INTERPOLATED PES}

Constructing an ab initio PES is an expensive procedure even for the smallest molecules. In practice, one often uses an interpolated surface $V_{\text {appr }}:=$ $V_{\text {interpol }}$. Namely, the accurate ab initio potential $V_{\text {acc }}$ is evaluated only on a sparse grid, and $V_{\text {appr }}$ is obtained by an interpolation from this grid. It is straightforward to estimate the "static" effect of such interpolation, e.g., by computing the upper bound $\Delta V_{\max }:=\max \left|V_{\text {interpol }}-V_{\text {acc }}\right|$ for the difference between the two potentials in the dynamically accessible region of the PES. However, it is difficult to predict, a priori, what will be the effect on dynamical observables. To answer this question, we consider $V_{\text {acc }}=V_{\text {Morse }}$ for $\mathrm{H}_{2}$ and $V_{\text {appr }}=V_{\text {interpol }}$ to be a PES interpolated from the Morse potential sampled only on a grid of 32 or 1,024 points.

Figures 5 and 6 compare the quantum vibrational dynamics on $V_{\text {interpol }}$ and $V_{\text {Morse }}$. We consider both a sparse interpolation (with 32 grid points, with $\Delta V_{\max }=0.033$ a.u., Fig. 5) and a dense interpolation (with 1,024 grid points and $\Delta V_{\max }=3.5 \times 10^{-5}$ a.u., Fig. 6). Panels (a) of Figures 5 and 6 show the fidelity for the two cases, computed by DR with 512 trajectories and by the exact QM dynamics. Figures 5(a) and 6(a) show that the sparser grid leads to a rapid decay of fidelity, whereas the denser grid results in fidelity
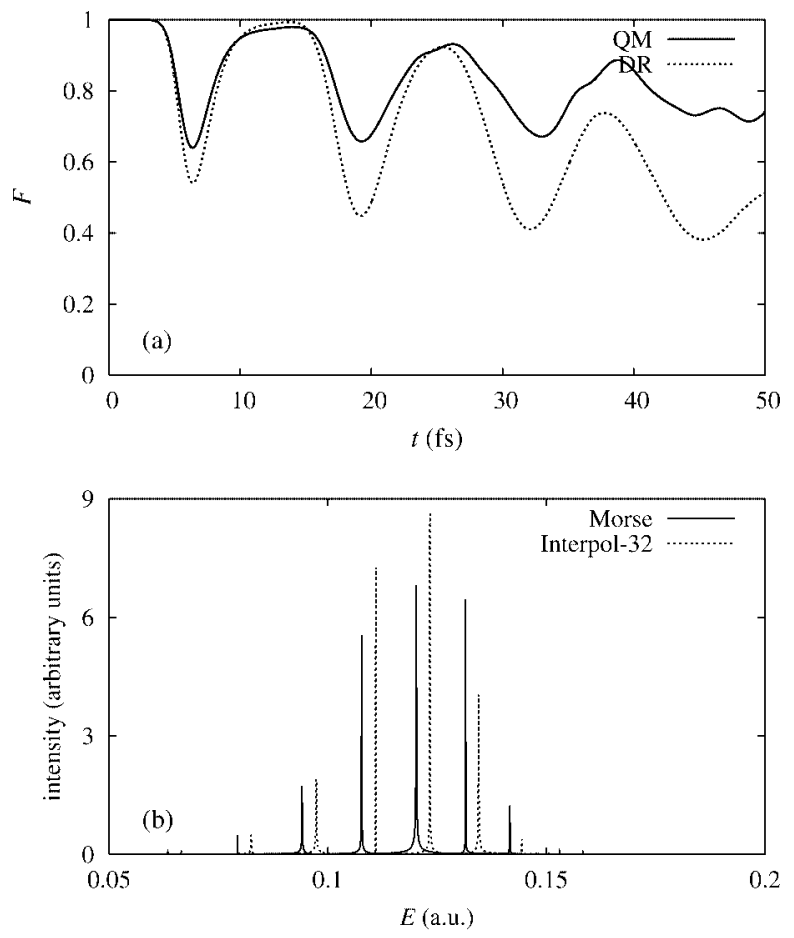

FIGURE 5. Comparison of vibrational quantum dynamics of $\mathrm{H}_{2}$ on $V_{\text {Morse }}$ and the interpolated surface $V_{\text {interpol-32. }}$ The initial state was a Gaussian wave packet with $R_{0}=3$ a.u., $P_{0}=0$, and $\sigma_{r}=0.3$ a.u. (a) Quantum fidelity computed by the DR and exactly (QM). (b) Comparison of vibrational spectra of $V_{\text {interpol-32 }}$ and $V_{\text {Morse }}$.

very close to unity up to $t=50 \mathrm{fs}$. In both cases, fidelity is quite well predicted by the DR. Therefore, we expect that in the first case, the interpolation will have a large effect on the spectrum, whereas in the second case, the effect should be small. These predictions are confirmed in Figures 5(b) and 6(b) in which the spectra of $V_{\text {Morse, }}, V_{\text {interpol-32, }}$ and $V_{\text {interpol-1024 }}$ are compared. While the spectrum of $V_{\text {interpol-32 }}$ is very different from that of $V_{\text {Morse, }}$ the spectra of $V_{\text {Morse }}$ and $V_{\text {interpol-1024 }}$ are barely distinguishable.

Finally, we also analyzed the effects of interpolation of the ${ }^{1} \Pi$ excited PES of $\mathrm{CO}_{2}$. For this purpose, we computed the DR of fidelity between an interpolated and on-the-fly evaluated LEPS PESs. The interpolated LEPS PES was discretized and interpolated in the same way as the EOM-CCSD PES in Section 3.2. The DR of fidelity stayed above 0.97 until the completion of the first two and most important recurrences in the autocorrelation function and remained above 0.86 for the whole length of simulation (180 fs). As the LEPS PES is sufficiently similar 

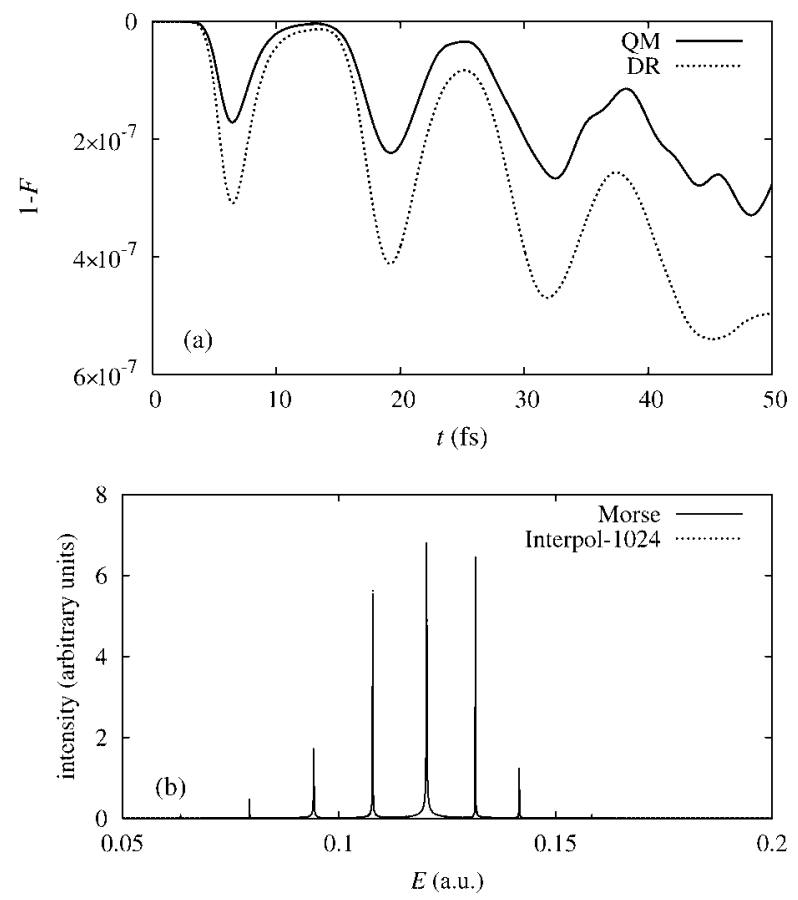

FIGURE 6. Comparison of vibrational quantum dynamics of $\mathrm{H}_{2}$ on $V_{\text {Morse }}$ and the interpolated surface $V_{\text {interpol-1024. }}$. The initial state was a Gaussian wave packet with $R_{0}=3$ a.u., $P_{0}=0$, and $\sigma_{r}=0.3$ a.u. (a) Quantum fidelity computed by the DR and exactly (QM). (b) Comparison of vibrational spectra of $V_{\text {interpol-1024 }}$ and $V_{\text {Morse. }}$

to the EOM-CCSD PES, we expect a similar effect of interpolation on the EOM-CCSD PES. Because of the relatively high value of fidelity, we expect the effect of the interpolation on the results of Section 3.2 to be very small.

\section{Conclusions}

In this article, dephasing representation of quantum fidelity was used to analyze the accuracy of quantum dynamics on an approximate analytical or interpolated PES in comparison with the dynamics on an ab initio PES. In all cases studied, the fidelity computed with the DR method proved to be a reliable estimate of the quantum fidelity. The method is easy to implement; in addition to a classical molecular dynamics code, it only requires the calculation of the action due to the difference between the two PESs. Nevertheless, the main advantage of the method is its efficiency. In case of the twodimensional model of $\mathrm{CO}_{2}$, the DR method is $\sim 100$ times faster than the quantum calculation. Furthermore, in contrast to quantum dynamics, the DR does not scale exponentially with the number of DOF and therefore can be easily applied to multidimensional problems.

In the future, we plan to perform exactly such a many-dimensional calculation, but in that case, it will be impossible to have a comparison with the exact quantum dynamics. Therefore, further work is needed to predict situations where the DR may fail. E.g., we have observed that the DR cannot reproduce recurrences in fidelity. However, these usually occur after fidelity decays to zero first, and then it is obvious that the approximate PES is insufficient.

In summary, the DR is a tool capable of estimating the accuracy of the quantum dynamics on an approximate PES without actually running the quantum dynamics simulation and without the need for a PES grid. Hence, the DR can be used during the initial phase of a quantum simulation to decide whether the method chosen to compute a PES allows for a dynamics of the desired accuracy. Or, as demonstrated in the last part of this article, the method can be used as a quick tool to find a sufficient grid point density for an ab initio PES.

\section{ACKNOWLEDGMENTS}

The authors thank Marius Wehrle for discussions.

\section{References}

1. Clary, D. C. Science 2008, 321, 789.

2. Makri, N. Annu Rev Phys Chem 1999, 50, 167.

3. Rabitz, H. Chem Rev 1987, 87, 101.

4. Li, B.; Mollica, C.; Vaníček, J. J Chem Phys 2009, 131, 041101.

5. Sato, S. J Chem Phys 1955, 23, 592.

6. Kulander, K. C.; Light, J. C. J Chem Phys 1980, 73, 4337.

7. Zimmermann, T.; Vaníček, J. J Chem Phys 2010 (in press).

8. Peres, A. Phys Rev A 1984, 30, 1610.

9. Gorin, T.; Prosen, T.; Seligman, T. H.; Žnidarič, M. Phys Rep 2006, 435, 33.

10. Vaníček, J. Phys Rev E 2004, 70, 055201(R).

11. Vaníček, J. Phys Rev E 2006, 73, 046204.

12. Cerruti, N. R.; Tomsovic, S. Phys Rev Lett 2002, 88, 054103.

13. Vaníček, J.; Heller, E. J. Phys Rev E 2003, 68, 056208.

14. Grebogi, C.; Hammel, S. M.; Yorke, J. A.; Sauer, T. Phys Rev Lett 1990, 65, 1527.

15. Miller, W. H.; Smith, F. T. Phys Rev A 1978, 17, 939.

16. Feit, M. D.; Fleck, J. J. A.; Steiger, A. J Chem Phys 1982, 47, 412 . 
17. Press, W. H.; Teukoldy, S. A.; Vetterling, W. T.; Flannery, B. P. Numerical Recipes in C; Cambridge University Press: Cambridge, UK, 1992.

18. Verlet, L. Phys Rev 1967, 159, 98.

19. Thom, J.; Dunning, H. J Chem Phys 1989, 90, 1007.

20. Morse, P. M. Phys Rev 1929, 34, 57.

21. Frisch, M. J.; Trucks, G. W.; Schlegel, H. B.; Scuseria, G. E.; Robb, M. A.; Cheeseman, J. R.; Scalmani, G.; Barone, V.; Mennucci, B.; Petersson, G. A.; Nakatsuji, H.; Caricato, M.; Li, X.; Hratchian, H. P.; Izmaylov, A. F.; Bloino, J.; Zheng, G.; Sonnenberg, J. L.; Hada, M.; Ehara, M.; Toyota, K.; Fukuda, R.; Hasegawa, J.; Ishida, M.; Nakajima, T.; Honda, Y.; Kitao, O.; Nakai, H.; Vreven, T.; Montgomery, Jr., J. A.; Peralta, J. E.; Ogliaro, F.; Bearpark, M.; Heyd, J. J.; Brothers, E.; Kudin, K. N.; Staroverov, V. N.; Kobayashi, R.; Normand, J.; Raghavachari, K.; Rendell, A.; Burant, J. C.; Iyengar, S. S.; Tomasi, J.; Cossi, M.; Rega, N.; Millam, J. M.; Klene, M.; Knox, J. E.; Cross, J. B.; Bakken, V.; Adamo, C.; Jaramillo, J.; Gomperts, R.; Stratmann, R. E.; Yazyev, O.; Austin, A. J.; Cammi, R.; Pomelli, C.;
Ochterski, J. W.; Martin, R. L.; Morokuma, K.; Zakrzewski, V. G.; Voth, G. A.; Salvador, P.; Dannenberg, J. J.; Dapprich, S.; Daniels, A. D.; Farkas, O.; Foresman, J. B.; Ortiz, J. V.; Cioslowski, J.; Fox, D. J. Gaussian 09, Revision A.02., Gaussian, Inc.: Wallingford, CT, 2009.

22. Schinke, R.; Engel, V. J Chem Phys 1990, 93, 3252.

23. VanVoorhis, T.; Heller, E. J. Phys Rev A 2002, 66, 050501(R).

24. Monkhorst, H. J. Int J Quantum Chem 1977, 12, 421.

25. Koch, H.; Jorgensen, P. J Chem Phys 1990, 93, 3333.

26. Stanton, J. F.; Bartlett, R. J. J Chem Phys 1993, 98, 7029.

27. Knowles, P. J.; Rosmus, P.; Werner, H.-J. Chem Phys Lett 1988, 146, 230.

28. Boys, S. F.; Bernardi, F. Mol Phys 1970, 19, 553.

29. Yoshino, K.; Esmond, J. R.; Sun, Y.; Parkinson, W. H.; Ito, K.; Matsui, T. J Quant Spectrosc Radiat Transf 1996, $55,53$.

30. Kuo, C. T.; Chen, Y. M.; Wang, S. Y.; Li, S. C.; Nee, J. B. Chinese J Phys 2004, 42, 65. 